\title{
LA AUTOGESTIÓN DE LOS APRENDIZAJES. INNOVACIÓN EN ESTRATEGIAS Y TECNOLOGÍAS. EL CASO DE ESTUDIANTES UNIVERSITARIOS DE LA FACULTAD REGIONAL SAN NICOLÁS
} SELF-MANAGED LEARNING. INNOVATION IN STRATEGIES AND TECHNOLOGIES. THE CASE OF UNIVERSITY STUDENTS FROM FACULTAD REGIONAL SAN NICOLÁS

\author{
Humberto Riccomi ${ }^{25}$ \\ Lucía Sacco ${ }^{26}$
}

\begin{abstract}
Resumen
Este artículo aporta conocimiento sobre una propuesta de enseñanza que favorezca la autogestión de los aprendizajes, implementada durante el 2018 en la que participaron 17 estudiantes de segundo año de Análisis Matemático II de la carrera de Ingeniería Electrónica de la Facultad Regional San Nicolás, UTN. La propuesta incluyó diferentes actividades de aprendizajes con el propósito de analizar los desempeños de los estudiantes frente a una metodología de trabajo diferente. El análisis de los resultados permitió comprender este tipo de escenario y de la necesidad de fortalecer el desarrollo de la autogestión de los aprendizajes en estudiantes de Ingeniería desde los primeros años.
\end{abstract}

Palabras claves: Autogestión. Estrategias. Tecnologías.

\begin{abstract}
This article provides knowledge about the implementation in 2018 of a teaching method which contributes to self-managed learning. Seventeen second-year Mathematical Analysis II students doing the degree in Electronic Engineering at Facultad Regional San Nicolás (FRSN), UTN, participated. The method included several learning activities in order to analyze the performance of students when faced to a different methodology of work at FRSN. The analysis of results contributed to the understanding of this teaching method and the need to strengthen the development of self-managed learning in engineering students during the first years.
\end{abstract}

Key words: Self-management. Strategies. Technologies.

25 Ingeniero eléctrico. Especialista en Docencia Universitaria. Profesor Asociado. Investigador categorizado Plan de Incentivos (categoría IV). Facultad Regional San Nicolás, Buenos Aires. Universidad Tecnológica Nacional. Argentina. E-mail: hriccomi@peeirr.com.ar

26 Lic. en Ciencias Aplicadas. Lic. Tecnología Educativa. Magister en Docencia Universitaria. Profesor Adjunto. JTP. Investigador categorizado Plan de Incentivos (categoría IV). Director Proyecto investigación PID Facultad. Facultad Regional San Nicolás, Buenos Aires. Universidad Tecnológica Nacional. Argentina. E-mail: Icsacco@gmail.com 


\section{INTRODUCCIÓN}

La enseñanza en la Universidad no debe considerarse como el resultado de la transmisión de conocimientos de un docente a un estudiante pasivo, sino debe concebirse como el logro productivo del estudiante que aprende en interacción social con otros estudiantes, con el docente y con el material didáctico que se le propone (Riccomi, Sacco \& Pacini, 2017).El docente universitario debe estar atento y crear el escenario adecuado que proponga caminos que despierten el desarrollo de cada potencial individual, generando aprendizaje de conocimiento, no sólo a nivel cognitivo, sino de actitudes, valores y funciones que permitan al estudiante de carreras de Ingeniería, comprender y dar respuesta a los problemas y situaciones de la vida. Es intención del docente universitario preparar al estudiante para que logre ser un ingeniero competente (Riccomi, Schivo, Sacco, Pacini, López, 2015).

Este artículo presenta una experiencia piloto que tiene el propósito de contribuir con conocimiento en el contexto universitario, sobre un modelo pedagógico de aprendizaje híbrido que permita el desarrollo de estrategias de autogestión de los aprendizajes, relevantes en la enseñanza universitaria del siglo XXI. La propuesta de enseñanza en estudio se realizó en una de las unidades didácticas de la asignatura Análisis Matemático II (AMII) en Ingeniería Electrónica de la Facultad Regional San Nicolás, Universidad Tecnológica Nacional (FRSN-UTN) durante el 2018.

\section{ENCUADRE TEÓRICO}

La innovación educativa debe visualizarse desde una perspectiva integral, donde la combinación de estrategias didácticas y recursos tecnológicos adecuados y un nuevo modo de analizar el rol del docente y el desempeño del estudiante, lleve a la construcción real de aprendizajes significativos y a la formación de competencias. Los modelos pedagógicos de aprendizaje híbrido (blended learning, blearning) están surgiendo como una innovación educativa, como una posibilidad de compromiso estudiantil, innovación sostenible en comparación con el aula tradicional. Estos modelos pedagógicos están cada vez más utilizados en la educación superior, por el hecho que promueven cambios significativos en el aprendizaje y brindan la posibilidad de crear nuevos escenarios que se adapten a las necesidades particulares de cada estudiante. La integración de la enseñanza tradicional presencial, con el sistema e-learning, permite crear modelos educativos flexibles y acordes a los nuevos tiempos, con el propósito de no renunciar a las posibilidades que ofrecen ambos (Dziuban \& Hartman, 2004; Graham, 2006).

Duart, Gil, Pujol \& Castaño (2008) hacen una distinción importante entre los conceptos blended e híbrido. Para este autor, en el resultado de la mezcla (blended) se pueden distinguir las partes que la componen, en cambio el concepto híbrido es el resultado del cruce de dos elementos de origen diferenciado, cuyo resultado está totalmente integrado e inseparable. De esta manera, el concepto híbrido constituye una posibilidad de continuo en los procesos de enseñanza y de aprendizaje puesto que puede verse como la expansión y continuidad espacio temporal (presencial y no presencial, sincrónica y asincrónica) en el ambiente de aprendizaje. Los ambientes híbridos van más allá del complemento de la presencialidad con la virtualidad o viceversa, se trata de la integración de ambas modalidades, proporcionando dinámicas y espacios de aprendizaje dialógico, que replantean los roles del estudiante, del profesor y del proceso de aprendizaje.

La autogestión del aprendizaje es la acción de activar y mantener sistemáticamente procesos metacognitivos, motivacionales, afectivos y conductuales, con el fin de alcanzar objetivos de aprendizaje, en un contexto en particular. Una propuesta de enseñanza basada en un modelo pedagógico de aprendizaje híbrido debe pensarse en un escenario que permita dicha autogestión del aprendizaje del estudiante (Zimmerman, 2008).

Para Chávez Barboza (2014), la autogestión de los aprendizajes implica cuatro fases distintivas, la planificación, el seguimiento, el control y la evaluación. En cada una de ellas el estudiante debe identificar sus propias necesidades, establecer sus propios objetivos de aprendizaje, realizar búsquedas de recursos (humanos y materiales), elegir e implementar sus propias estrategias y métodos, y realizar actividades de evaluación de los resultados.

Villardón Gallego (2007) señala que es importante que el curriculum universitario ayude a los estudiantes a convertirse en personas que gestionen sus propios recursos para aprender. Para este autor la enseñanza en el contexto universitario debe estar orientada al desarrollo integral del estudiante, y debe incluir diferentes 
acciones para desarrollar la competencia para aprender. Dentro de esta competencia consideran la autonomía y la capacidad de gestionar el propio aprendizaje y las actuaciones destinadas a favorecerla.

Soler Ranzani, Solanas Saura, Aymerich Martínez \& Brugada Terradellas (2011) resaltan la importancia de una buena planificación, organización y capacidad resolutiva, tanto por parte del estudiante como del docente. Se requiere que el estudiante se haga consciente de que debe aceptar responsabilidades para su aprendizaje y la necesidad de aprender por sí solo y de sus compañeros en forma cooperativa. Por otro lado, señalan que para que se desarrollen estos procesos en los estudiantes, la propuesta de enseñanza debe considerar una intervención continua del docente, tanto jerárquica como facilitadora.

Cuando se habla de competencias se ha acordado en la cátedra de Análisis Matemático II, considerar la definición dada por el Consejo Federal de Decanos de Ingeniería de la República Argentina:

Competencia es la capacidad de articular eficazmente un conjunto de esquemas (estructuras mentales) y valores, permitiendo movilizar (poner a disposición) distintos saberes, en un determinado contexto con el fin de resolver situaciones profesionales. (CONFEDI, 2014, p.16)

Según esta definición, las competencias aluden a capacidades complejas e integradas; están relacionadas con saberes (teórico, contextual y procedimental); se vinculan con el saber hacer (formalizado, empírico, relacional); están referidas al contexto profesional (entendido como la situación en que el profesional debe desempeñarse o ejercer); están referidas al desempeño profesional que se pretende (entendido como la manera en que actúa un profesional técnicamente competente y socialmente comprometido); permiten incorporar la ética y los valores.

\section{OBJETIVO}

Desde este posicionamiento, los docentes de la cátedra de AMII se proponen, a partir del diseño y puesta en acción de la propuesta de enseñanza piloto:

- Contribuir con conocimiento en el contexto de la FRSN-UTN, sobre un modelo pedagógico de aprendizaje híbrido que permita el desarrollo de competencias y la autogestión de los aprendizajes por parte de los estudiantes de 2 do año que cursan AMII en Ingeniería Electrónica.

\section{ENCUADRE METODOLÓGICO}

A partir de la intencionalidad de los docentes de la cátedra, una propuesta de enseñanza que facilite un aprendizaje híbrido de calidad debería incluir:

- Un escenario apropiado, flexible, que permita la actuación, la experimentación y el error y, sobre la base de las producciones de los estudiantes, creando oportunidades de intercambio y reflexión.

- Métodos variados que resulten apropiados para la adquisición de aprendizajes: conceptos y teorías, competencias, habilidades y valores.

- Actividades centradas en el estudiante, ajustadas al tiempo y recursos disponibles, que permita la gestión de sus propios ritmos de trabajo y estudio.

- Prácticas y herramientas que permitan llevar a cabo un proceso dialógico entre docentes, estudiantes y recursos.

La hipótesis de estudio formulada fue:

Una propuesta de enseñanza que facilite el aprendizaje híbrido contribuye a mejorar el desarrollo de competencias específicas vinculadas con la autogestión de los aprendizajes de estudiantes de 2 do año de Análisis Matemático II, de la carrera de Ingeniería Electrónica de la Facultad Regional San Nicolás, UTN.

La investigación realizada adoptó un enfoque cualitativo ya que pretendió comprender y analizar el impacto de la implementación de una propuesta de enseñanza basada en un modelo de aprendizaje híbrido. El estudio del diseño y la implementación de la propuesta se realizó desde la perspectiva de los estudiantes y teniendo en cuenta las percepciones de los docentes. 
Su alcance fue descriptivo, ya que buscó detallar los momentos de aprendizaje por los que transcurrió el estudiante al relacionarse con cada una de las actividades de aprendizaje propuestas, analizando cómo las mismas influyeron en el desarrollo de competencias genéricas y específicas.

El tipo correspondió a un estudio de casos, ya que se analizó en profundidad el grupo-clase durante la enseñanza de una unidad didáctica. El diseño fue transversal porque se recolectaron datos en un momento determinado, durante la implementación de la propuesta de enseñanza, para el estudio de las competencias de autogestión de aprendizaje que desarrollen los estudiantes.

Se trabajó con dos grupos de estudiantes, un grupo experimental conformado por 17 estudiantes de 2 do año de la carrera de Ingeniería Electrónica y un grupo control de 14 estudiantes de 2do año de Ingeniería Eléctrica que cursaron durante el mismo período la misma asignatura.

\section{PROPUESTA DE ENSEÑANZA}

En primer lugar, se analizó en qué unidades se iba a proponer este modelo de enseñanza y con qué grupo de estudiantes. Análisis Matemático Il es una asignatura anual, organizada en torno a tres unidades didácticas. La última unidad didáctica del año lectivo, es la de Ecuaciones y sistemas de ecuaciones diferenciales, constituida por cuatro sub unidades. Las clases son presenciales, dos veces en la semana, de tres horas cada una. Tradicionalmente esta unidad didáctica se evalúa a través de un parcial teórico-práctico.

Durante el 2018 se consideró que los contenidos incluidos en ella permitirían llevar a cabo una experiencia piloto en la FRSN que proponga una dinámica de trabajo activo, tanto de docentes y estudiantes, alternando instancias presenciales y no presenciales.

La Tabla 1 presenta las competencias y resultados de aprendizajes fijados por la cátedra, para la unidad didáctica seleccionada.

\section{Tabla 1}

Competencias y resultados de aprendizaje

\begin{tabular}{|c|c|c|}
\hline \multicolumn{2}{|l|}{ Competencias } & \multirow{2}{*}{$\begin{array}{l}\text { Resultados de aprendizaje } \\
\text { Modelice y resuelva problemas de } \\
\text { aplicación utilizando conceptos de AMII } \\
\text { (EDO, tipos de funciones, ...) }\end{array}$} \\
\hline GENÉRICAS & $\begin{array}{l}\text { Identificar, formular y resolver } \\
\text { problemas de ingeniería electrónica }\end{array}$ & \\
\hline & & $\begin{array}{l}\text { Visualice e interprete soluciones de los } \\
\text { problemas modelizados }\end{array}$ \\
\hline & $\begin{array}{l}\text { Utilizar de manera efectiva las técnicas } \\
\text { y herramientas de aplicación en la } \\
\text { ingeniería electrónica. }\end{array}$ & $\begin{array}{l}\text { Aplique correctamente los conocimientos } \\
\text { teóricos a la práctica }\end{array}$ \\
\hline & Comunicar con efectividad. & $\begin{array}{l}\text { Argumente razonamientos y toma de } \\
\text { decisiones }\end{array}$ \\
\hline & $\begin{array}{l}\text { Aprender en forma continua y } \\
\text { autónoma. }\end{array}$ & $\begin{array}{l}\text { Valore las instancias de aprendizaje fuera } \\
\text { del ámbito de la clase }\end{array}$ \\
\hline & Actuar con espíritu emprendedor. & $\begin{array}{l}\text { Autoevalue permanentemente fortalezas, } \\
\text { debilidades y potencialidades }\end{array}$ \\
\hline $\begin{array}{l}\text { ESPECÍFICA } \\
\text { DE LA UNIDAD } \\
\text { DIDÁCTICA DE } \\
\text { AMII }\end{array}$ & $\begin{array}{l}\text { Formular modelos matemáticos de } \\
\text { situaciones problemáticas vinculadas } \\
\text { a fenómenos físicos, químicos o } \\
\text { geométricos a través de ecuaciones y } \\
\text { sistemas de ecuaciones diferenciales }\end{array}$ & $\begin{array}{l}\text { Modelice y resuelva, a través de } \\
\text { ecuaciones y sistemas de ecuaciones } \\
\text { diferenciales, situaciones problemáticas } \\
\text { vinculadas a fenómenos físicos, químicos o } \\
\text { geométricos. }\end{array}$ \\
\hline
\end{tabular}

Fuente: Elaboración propia 
Entre las barreras anticipadas al desarrollo de la propuesta de enseñanza se tuvo en cuenta problemas en la conectividad de los estudiantes y la disponibilidad de recursos tecnológicos, los escasos horarios disponibles de los laboratorios en la FRSN y las posibilidades en cuanto a permisos para la implementación de la propuesta por parte de las autoridades de la Facultad. Como escenario de trabajo se consideró diseñar y desarrollar un Aula Virtual en la plataforma Moodle de la FRSN-UTN. Este proceso implicó para los docentes un arduo trabajo de reflexión sobre la manera óptima de organización y secuenciación de las actividades a proponer, como así también, de los recursos a incluir para el desarrollo de la teoría, el estudio de procedimientos de resolución de ejercicios, la atención de dudas y consultas o para ampliar conocimientos sobre el contenido a estudiar en forma autónoma por los estudiantes propuestos en las clases no presenciales.

La experiencia se inició el 02 de octubre y se finalizó el 17 de noviembre de 2018. Constó de 5 clases presenciales, 9 clases no presenciales y 4 sesiones de Zoom.

Dos semanas antes de iniciada la experiencia, se les asignó a los estudiantes la realización de un test de estilos de aprendizajes validado on line (Felder \& Silverman, 2002). Se le pidió una vez realizado enviar los resultados a los docentes. Este modelo de test establece que los estudiantes aprenden de diferentes maneras. Hay estudiantes que prefieren escuchar u observar; reflexionar o actuar; razonar de manera lógica o intuitiva; memorizar o visualizar y establecer analogías; de manera constante o en pequeños trozos y en forma global, es decir, poseen preferencias de aprendizaje en una dimensión: sensorial o intuitiva, visual o verbal, activo o reflexivo, secuencial o global respectivamente (Felder \& Spurlin, 2005). Propone la hipótesis de que los docentes de Ingeniería deberían considerar, al diseñar y plantear un modelo o estilo de enseñan$\mathrm{za}$, aspectos de ambas dimensiones de una categoría (por ejemplo, visual y verbal). Se consideró que este modelo proporcionaría una buena base para los docentes de la asignatura para realizar los ajustes finales al diseño del Aula Virtual antes de su implementación, acorde las necesidades de aprendizaje de cada uno de los estudiantes.

La primera actividad propuesta en el Aula Virtual fue la realización de un glosario colaborativo. Los estudiantes debían aportar un concepto, propiedad o procedimiento que consideraran que no ha sido contemplado en el Aula Virtual y que ellos han necesitado para la realización de las tareas.

El Aula Virtual presentaba la unidad didáctica dividida en cuatro secciones, cada una correspondiente a una de las sub unidades que la conforman. Cada sección se estructuró de una primera clase presencial y una a tres clases no presenciales. Todas las clases presenciales se desarrollaron en el salón de clases. Los estudiantes asistían a ellas con sus propias computadoras portátiles. En las clases presenciales se realizaba un recorrido y explicación sobre las tareas asignadas. Estas tareas hacían referencia a desarrollos de teoría, modelización y resolución de problemas, trabajo con el software Geogebra o con las aplicaciones de Matlab desarrolladas por la cátedra, o la resolución de ejercicios incluidos en el cuadernillo propio de la asignatura.

El estudiante podía llevar a cabo las clases no presenciales en el espacio y tiempo que considerara, ya sea utilizando los laboratorios de la facultad o desde sus hogares. Los docentes en el horario de clases, incluían preguntas en los foros de consultas de tareas que orientaban el estudio de los contenidos de la clase no presencial, así como acompañaban el trabajo autónomo de los estudiantes.

Para la atención puntual de cada una de las cuatro entregas de tareas, como así también para fomentar la oralidad y el intercambio de dudas, se realizaron sesiones de Zoom. Los estudiantes durante estas reuniones on-line presentaban dudas en forma oral. Los audios y chat producidos en ellas luego eran compartidos en el Aula Virtual, para su posterior uso por parte de quien lo necesitara, ya sea porque no pudo asistir a la reunión o para rever lo analizado.

Otra de las acciones que se propuso a los estudiantes es el uso de bibliografía específica de AMII. En cada una de las tareas se presentaba bibliografía sugerida disponible en la Biblioteca de la FRSN, teniendo cada uno la libertad de buscar otras fuentes, debidamente referenciadas y de rigor científico. A partir del trabajo realizado por los estudiantes a lo largo de la experiencia se fueron ajustando consignas y se incorporó bibliografía específica digitalizada.

Para la recuperación, de aquellas tareas que a lo largo de la experiencia no habían sido realizadas satisfactoriamente por parte de los estudiantes, se propusieron en forma personalizada nuevas tareas de recuperación.

Los dos docentes a cargo de la experiencia realizaron diferentes actividades de intervención vinculadas con: 
- La dirección de la propuesta: dirigiendo y marcando el paso de trabajo con los contenidos y fijando días de entrega de tareas.

- El acompañamiento de los estudiantes: respondiendo consultas puntuales y generales, aportando ayudas extras para la interpretación de contenidos o de consignas (nuevos recursos), interrelacionando conocimiento de temas para la discusión a medida que iba transcurriendo la propuesta, ayudando a clarificar las ideas y el conocimiento de los estudiantes solicitando opiniones, corrigiendo intervenciones de los estudiantes, evaluando la actuación de los estudiantes, intercambiando ideas y experiencias con los estudiantes.

- La gestión de instancias de confrontación: proponiendo debates a través de preguntas que permitieran a los estudiantes manifestar posibles tareas o actividades que le generaban conflictos (principalmente en las sesiones de Zoom), identificando posibles errores cometidos por los estudiantes para poder intervenir adecuadamente, discutiendo y negociando estrategias de tutorías con los estudiantes.

- La gestión de instancias de reconocimiento del desafío de aprender en forma autónoma: aclarando ideas identificando contradicciones en las contestaciones de estudiantes, promoviendo la evaluación de ideas y el pensamiento crítico, despertando el interés de los estudiantes relacionando la discusión con conocimientos o aprendizajes previos, estimulando a que los estudiantes aprendan por sí mismos a través del descubrimiento y la exploración.

El seguimiento de los estudiantes a lo largo de toda la experiencia, permitió a los docentes de la asignatura valorar el trabajo de autogestión de los aprendizajes que cada uno de los estudiantes llevó a cabo. Entre los aspectos relevantes observados:

- La motivación intrínseca, interés por el propio aprendizaje que cada estudiante demostró a lo largo de la experiencia para superar las dificultades de un modo de trabajo al que no estaban familiarizados.

- La motivación extrínseca, interés por la realización completa de las tareas y actividades propuestas (lo cual implicaba la no realización del Tercer Parcial escrito individual presencial).

- La autoeficacia para trabajar con recursos tecnológicos (actividades de construcción colaborativa y de comunicación sincrónica y asincrónica). En las sesiones de Zoom mostraron ser nativos digitales en el uso de dicha tecnología, asistiendo en varias oportunidades en cuestiones técnicas de uso a los docentes como compartir pantalla o guardar el chat de conversación cuando uno de los estudiantes no tenía micrófono.

- La elaboración de esquemas o desarrollos propios, o de organización estableciendo conexiones entre los contenidos de las unidades o los procedimientos de resolución.

- El desarrollo de un pensamiento crítico, argumentando las soluciones de los problemas propuestos, vinculándolos con lo trabajado en otras asignaturas, interpretando soluciones obtenidas no sólo desde el análisis del resultado desde su representación gráfica y su vinculación con el problema físico.

- La regulación metacognitiva del propio aprendizaje, planificando tiempos para el desarrollo de las tareas, realizando análisis de las estrategias de estudio utilizadas, evaluando los propios avances y corrección del proceso llevado a cabo.

- La gestión individual del ambiente de estudio. Muchos alumnos evidenciaron a un principio ciertas dudas en cuanto a cómo organizar las condiciones adecuadas del espacio de trabajo no presencial para estudiar y la gestión eficaz del tiempo. Los docentes lo evidenciaron en un principio, por mensajes desesperados a través del WhatsApp del grupo de clases. Ello fue mejorando a partir de la segunda sección, una vez superado los temores de entrega de tareas y recibo de devoluciones por parte de los docentes.

- El apoyo entre compañeros para la resolución de las tareas, aspecto trabajado durante las devoluciones de las primeras tareas. Se alentó el trabajo colaborativo y la construcción de sus propios desarrollos, interpretaciones y producciones.

- La participación en foros de consulta individual (Aula Virtual o WhatsApp) y sesiones de consulta grupal (de Zoom).

La evaluación de las ocho tareas asignadas a los estudiantes se realizó a partir de diferentes rúbricas que permitieron valorar el alcance de los resultados de aprendizajes fijados para la unidad didáctica en cuanto 
al desarrollo de teoría, el uso de los recursos tecnológicos propuestos, la resolución de problemas y la realización de ejercicios.

\section{RESULTADOS}

Los resultados obtenidos del análisis de los estilos de aprendizajes de los estudiantes de Análisis Matemático II resultaron de mucha utilidad. El 50\% del grupo de 17 estudiantes del grupo experimental resultaron ser activos (puntaje promedio de la dimensión 4,6 ) y el otro $50 \%$ reflexivos (puntaje promedio de la dimensión 2,5). El 81\% del grupo de 17 estudiantes resultaron sensitivos y el otro19\% intuitivos. Los resultados mostraron una fuerte tendencia de estudiantes visuales (75\%). Por último, los resultados muestran una tendencia de estudiantes secuenciales (69\%).

Toda esta información permitió la toma de decisiones curriculares, pedagógicas y tecnológicas (Mishra \& Koehler, 2015). Decisiones tomadas a partir interrogantes como ¿Qué tipo de información enfatizar en el Aula Virtual? ¿Priorizar procedimientos y aplicaciones o conceptos y teoría? ¿Qué modo de presentación hay que destacar? ¿Visual con cuadros, diagramas, videos, figuras) o verbales con foros, lecturas y discusiones? ¿Qué dinámica de trabajo debe proponer el Aula Virtual, que obedezca a un modelo pedagógico de aprendizaje híbrido, potencie la autogestión de los aprendizajes? ¿Qué intervención docente se debe considerar? ¿De manera inductiva a través de fenómenos que conduzcan al desarrollo de teoría o deductivamente, partiendo de leyes y principios que conduzcan al estudio de problemas como el de un circuito eléctrico RLC serie con distintas alimentaciones o un sistema masa-resorte con rozamiento? ¿Qué modo de participación por parte de los estudiantes es facilitado por el uso del Aula Virtual? ¿Activos (los estudiantes hablan, discuten, comparten) o pasivos (estudiantes sólo observan y escuchan)? ¿Qué tipo de organización debe presentar el Aula Virtual? ¿Secuencial (paso a paso) o global (partiendo del todo)?

Como respuesta a estas preguntas, se consideró la incorporación de estrategias y tecnologías específicas. Para reforzar la verbalidad, se incluyeron los foros de consultas en cada una de las tareas; para trabajar la oralidad, la inclusión de las sesiones de Zoom; para aprovechar la predisposición a lo visual se incorporó el trabajo con videos tutoriales, con aplicaciones de Matlab propias y software de geometría dinámica (GeoGebra) para la representación de familias de curvas solución de las ecuaciones diferenciales, de isóclinas y trayectorias ortogonales.

Por último, para atender la predisposición de los estudiantes a aprender en forma secuencial se trató de presentar lo más detalladamente cada una de las secciones, atendiendo día a día posibles dificultades u obstáculos presentados por los estudiantes para el trabajo con el Aula Virtual.

De los 17 estudiantes del grupo experimental que iniciaron la experiencia, 15 fueron los que la completaron. Sólo un estudiante solicitó a los docentes cambiarse de comisión (con una enseñanza tradicional) y otro estudiante abandonó la experiencia. En el primer caso, el alumno aludió sentirse inseguro para realizar la experiencia, y el otro explicitó problemas en la disponibilidad de recursos tecnológico (luego se constató que adeudaba Análisis Matemático I y le resultaba dificultoso el seguimiento de la asignatura). Al finalizar la experiencia y comparar los resultados, sobre una base de 14 alumnos del GT y 17 alumnos en el GE, se tuvo que en el GT hubo 5 alumnos ausentes al momento de evaluar, 4 alumnos en condiciones de regularizar y 5 alumnos en condiciones de promoción directa, mientras que en el GE hubo 2 alumnos ausentes al momento de evaluar, 3 alumnos en condiciones de regularizar y 12 alumnos en condiciones de promoción directa.

Por último, y para tener un panorama general de todo el cursado, ambos grupos terminaron con 4 alumnos libres. En el GT, 6 alumnos quedaron en condición regular y 7 alumnos acreditaron la asignatura, mientras que, en el GE, 4 alumnos quedaron en condición regular y 12 alumnos acreditaron la materia.

Como cierre de la propuesta en el Aula Virtual se incluyó un formulario de Google preparado por los docentes de la cátedra, para la evaluación de la propuesta del modelo pedagógico de aprendizaje híbrido por parte de los estudiantes. En él se les consultó. por un lado, sobre la claridad de las consignas de las tareas, la organización y uso del Aula Virtual, de las dificultades para la realización de las tareas, de la utilidad de los recursos tecnológicos incluidos para el estudio de los contenidos (videos, aplicaciones, tutoriales, documentos, enlaces). Por otro lado, se los invitó a realizar una autoevaluación de resultados de aprendizaje que ellos consideraban alcanzados, contar una anécdota que les hubiera pasado durante la experiencia y realizar una opinión personal sobre la modalidad de trabajo llevada a cabo en Análisis Matemático II. 


\section{CONCLUSIONES}

Los resultados son alentadores, pero no se debe perder de vista de que se trata de una experiencia puntual, y que nuestra intención como integrantes de un grupo de investigación educativa, es continuar en esta línea para aumentar y consolidar los resultados obtenidos en este trabajo. La opinión de los estudiantes ha sido muy satisfactoria. Las sugerencias, comentarios, consultas y dudas planteadas por ellos, permitieron realizar ajustes a la propuesta de enseñanza a diario que permitieran generar ideas relativas a la importancia de estudiar, investigar y experimentar en estrategias y tecnologías que brinden a los estudiantes un rol activo en el proceso de aprendizaje.

Desde lo curricular y respecto al uso de las TIC, se considera continuar trabajando en el diseño del Aula Virtual, pensando e investigando nuevos problemas y recursos que permitan mayor autonomía en el trabajo que realicen los estudiantes, como así también habilidad para tomar decisiones acertadas en el momento oportuno, con una postura de reflexión permanente ante cambios posibles de la realidad que lo rodea, a transferir conocimiento a situaciones nuevas y a construir una postura que integre los aspectos cognitivos, las habilidades y el pensamiento crítico necesario para la autogestión de su aprendizaje. Desde lo didáctico, seguir reflexionando en cuanto a las estrategias de enseñanza y actividades de aprendizaje, las cuales estén direccionadas a desarrollar competencias profesionales y lograr un conjunto de aptitudes que permitan, a cada uno de los estudiantes, resolver situaciones problemáticas de interés a la especialidad, en diversos contextos, de manera independiente y flexible. El valor agregado de este tipo de trabajo, en una asignatura como es Análisis Matemático Il de segundo año del ciclo básico, es descubrir su implicancia en la Ingeniería.

\section{REFERENCIAS}

Chávez Barboza, E. (2014). Autogestión del aprendizaje en la investigación educativa sobre Entornos Personales de Aprendizaje (PLE): Una revisión de literatura. Revista de Educación Mediática y TIC, 3 (2), 114-134.

Consejo Federal de Decanos de Ingeniería (2014). Competencias en Ingeniería. Declaración de Valparaíso. Universidad FASTA. Mar del Plata. Argentina.

Duart, J. M.; Gil, M.; Pujol, M.; Castaño, J. (2008). La universidad en la sociedad red. Barcelona: UOC/Ariel. 1. a ed.

Dziuban, C.; Hartman, J. L..; Moskal, P. (2004). Blended learning. Educause Research Bulletin. Vol.7. Educause Center for Applied Research(ECAR).

Felder, R.; Silverman, L. (2002). Learning and teaching styles in engineering education. Journal of Engineering Education, 78 (7), 674-681.

Felder, R.; Spurlin, J. (2005). Reliability and validity of the Index of Learning Styles. A meta-analysis. International Journal of Engineering Education, 21(1), 103-112.

Mishra, P.; Koehler, M.; Cain, W. (2015). ¿Qué son los Saberes Tecnológicos y Pedagógicos del Contenido (TPACK)? Virtualidad, Educación y Ciencia. 6, 10.

Riccomi, H., Schivo, M. E., Sacco, L., Pacini, C., López, R. (2015). Desarrollo de competencias y formulación de intenciones educativas en Análisis Matemático II (p. 87-96). XIX EMCI Nacional y XI EMCI Internacional. Educación Matemática en Carreras de Ingeniería. San Nicolás. Buenos Aires. Argentina.

Riccomi, H., Sacco, L., Pacini, C. (2017). Mutando Prácticas Universitarias. Un transcurrir entre investigaciones e intenciones educativas para el logro de competencias profesionales. Saarbrücken, Alemania: Editorial Académica Española. Recuperado de: https://www.morebooks.de/store/gb/book/mutando-pr\%C3\%A1cticas-universitarias/ isbn/978-3-639-53510-5

Soler Ranzani, M.; Solanas Saura, P.; Aymerich Martínez, M. y Brugada Terradellas, R. (2011). Autogestión en el proceso de aprendizaje: el viaje a Itaca. Univest 2011. Universidad de Girona. Recuperado de: https://dugi-doc.udg.edu/ bitstream/handle/10256/3659/181.pdf?sequence=1

Villardon Gallego, L. (2015). Competencias genéricas en Educación Superior. Metodologías específicas para su Desarrollo. Madrid: Narcea, 232-233.

Zimmerman, B. J. (2008). Investigating self-regulation and motivation: Historical background, methodological development, and future prospects. American Educational Research Journal, 45(1), 166-183.

Fecha de recepción: 18/03/2019

Fecha de aceptación: 07/10/2019 